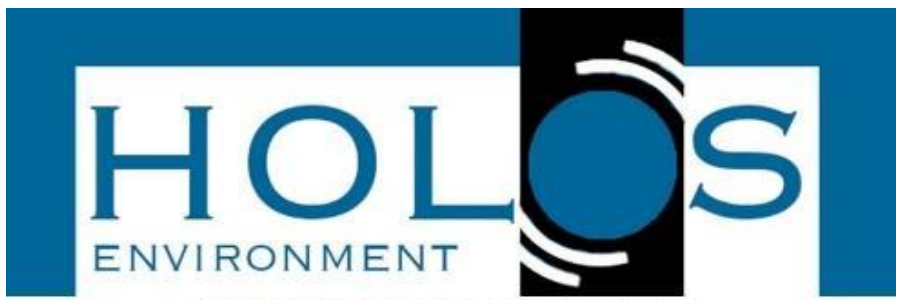

\title{
APLICATIVO WEB PARA CARACTERIZAR A QUALIDADE DA ÁGUA
}

\section{WEB APPLICATION FOR WATER QUALITY CHARACTERIZATION}

\author{
Rosa Alencar Santana de Almeida ${ }^{1}$; Caio Sacramento de Britto Almeida²; Lucas dos \\ Santos Souza²; Débora Abdalla Santos²
}

Artigo recebido em: 02/07/2020 e aceito para publicação em: 25/08/2020. DOI: http:/dx.doi.org/10.14295/holos.v21i1.12408

Resumo: Diante do atual cenário dos recursos hídricos, os índices de qualidade de água (IQA) são recursos apropriados para comunicar, ao público em geral, a qualidade da água de mananciais superficiais e subterrâneos. Os índices são formulações matemáticas agregadoras de parâmetros que resumem as informações em um número, permitindo uma leitura fácil ao público não técnico. Ao longo dos anos foram desenvolvidas várias expressões de IQAs, muitas com rigidez na escolha dos parâmetros indicadores de qualidade e/ou com limitações na região geográfica de aplicação. Foi elaborado um Índice de Qualidade da Água (e-IQUAS) que permite a escolha de parâmetros de maneira flexível, sem limite no número de variáveis e pode ser aplicado aos múltiplos usos da água. Para permitir seu uso irrestrito à sociedade, foram desenvolvidos, com ferramentas de software livre: um aplicativo web, amigável e fácil de usar, e uma rede social para disseminação de informações sobre o tema. $O$ aplicativo foi testado, verificado e está disponível na rede mundial de computadores. O trabalho contribui, portanto, para disseminar o uso do índice de qualidade de água e-IQUAS, de fácil aplicação e adaptado a qualquer região ou compartimento hídrico.

Palavras-chave: Índice de Qualidade de Água. Monitoramento. Aplicativo. Software Livre.

Abstract: In view of the current scenario of water resources, water quality indexes (WQI's) are appropriate tools to communicate, to the general public, the water quality of surface and groundwater sources. Indexes are mathematical formulations that aggregate parameters that summarize information into a number, allowing the non-technical public to read it easily. Over the years, several expressions of IQAs have been developed, many with rigidity in the choice of quality indicator parameters and/or with limitations in the applicable geographic region. A Water Quality Index (e-IQUAS) was developed that allows the choice of parameters flexibly, without limit on the number of variables, and can be applied to multiple uses of water. To allow its unrestricted use to society, free software tools were developed: a web application, friendly and easy to use, and a social network for the dissemination of information on the topic. The application has been tested, verified, and is available on the world wide web. The work, therefore, contributes to disseminating the use of the e-IQUAS water quality index, which is easy to apply and adaptable to any region or water storage.

Keywords: Water Quality Index. Monitoring. Application. Free Software.

\section{INTRODUÇÃO}

Os recursos hídricos têm importância fundamental na economia das nações; a

\footnotetext{
${ }^{1}$ Universidade Federal do Recôncavo da Bahia (UFRB), Cruz das Almas, BA. E-mail: rosaalencar@ufrb.edu.br

${ }^{2}$ Universidade Federal da Bahia (UFBA), Salvador, BA. E-mails: caiosba@gmail.com, lukas.santos15@gmail.com, abdalla@ufba.br
} 
demanda em todos os setores da vida produtiva tende a crescer, e a disponibilidade de água de boa qualidade tem diminuído. Estudos da Organização das Nações Unidas para a Educação, a Ciência e a Cultura evidenciam que o crescimento populacional e mudanças nos padrões de consumo, aliados ao desenvolvimento socioeconômico, concorrem com um aumento de cerca de $1 \%$ por ano na demanda de água desde a década de 1980, e assim deve continuar até 2050 (UNESCO, 2019).

A avaliação da qualidade de um compartimento hídrico é fundamental. A determinação da qualidade da água está relacionada a todos os ambientes com os quais a água esteve em contato em todo o ciclo hidrológico. Entretanto, como afirma Almeida (2012) os requisitos de qualidade são distintos para os diversos usos: uma massa de água pode ser adequada ao consumo humano, mas insatisfatória para uso em certo ramo da indústria; ou pode ser apropriada à dessedentação de animais e não indicada à irrigação. Segundo a Agência Nacional de Águas, a desatenção aos requisitos de qualidade da água para os vários usos representam um fator limitante para o seu aproveitamento (ANA, 2012).

Uma maneira de descrever a qualidade da água é relacionando, individualmente, as concentrações de todas as substâncias contidas em uma amostra. Como destaca Almeida (2012) esta relação tanto pode conter apenas os parâmetros mais comuns, como pode abranger até centenas de parâmetros variados; o que dificulta o entendimento. Assim, uma boa forma de relatar é utilizando os índices de qualidade de água. Para Abbasi, T. e Abbasi, S. (2012), quase todos os propósitos de caracterização da qualidade da água são adequados a algum tipo de índice: para auxiliar na tomada de decisões, para avaliar a qualidade da água em datas e em localizações geográficas diferentes, ou mesmo para manter o público informado sobre a qualidade de água de qualquer fonte e como suporte à pesquisa científica.

Os índices de qualidade de água são ferramentas utilizadas mundialmente. Várias formulações foram propostas e aprimoradas ao longo dos anos, algumas delas já disponibilizadas para cálculo via ambiente web. No site institucional do Canadian Council of Ministers of the Environment (CCME) estão disponíveis uma planilha eletrônica e um manual do usuário para cálculo do WQI CCME (CANADA, 2020) . Já - NSF Water Quality Index pode ser calculado por meio do aplicativo on-line disponibilizado pelo centro de pesquisas Water Reserch Center (WATER RESEARCH CENTER, 2020). 
Em geral, esses aplicativos computam índices com parâmetros e pesos previamente programados no aplicativo; não havendo funcionalidade para inclusão de novos parâmetros, o que exigiria alterações no código fonte do programa. Entretanto, como afirmam Abbasi, T. e Abbasi, S. (2012), o processo dinâmico da pesquisa científica enseja que os padrões sejam revisados continuamente: uma nova descoberta esclarece com novas evidências os efeitos benéficos ou prejudiciais de uma substância, ou revela dados sobre níveis de concentração que tornam um elemento prejudicial, ou a concentração abaixo da qual um elemento deixa de ser útil. $E$, portanto, são necessários aplicativos que contemplem estas exigências.

Este estudo recorre à formulação de um índice que oferece um método muito simples para descrever a qualidade da água, e apresenta o aplicativo e-IQUAS, desenvolvido com ferramentas computacionais da filosofia de software livre. O eIQUAS tem vantagens sobre índices pré-existentes, pois sua formulação, baseada na parametrização e flexibilidade na escolha dos parâmetros a serem amostrados, atende às necessidades de inserção ou supressão de substâncias para caracterizar a qualidade da água. Além disso, o e-IQUAS não tem limitações de compartimento hídrico e pode ser aplicado em qualquer região ou país. Para complementar, a pesquisa também inclui a criação de uma rede social, em plataforma web livre, para disseminação de trabalhos e informações sobre o tema.

\section{1 Índices de Qualidade de Água}

Esta seção é reservada à apresentação dos principais conceitos que norteiam a formulação dos índices de qualidade de água, e em particular o e-IQUAS:

\subsubsection{Premissas na formulação de índices}

Os índices de qualidade de água desenvolvidos até o final do século $X X$ notadamente acompanhavam a desatenção com a deterioração ambiental e não incorporavam a presença de substâncias químicas associadas a atividades humanas, responsáveis pela alteração da qualidade natural das águas (ALMEIDA, 2012). Na primeira década do século XXI, surgiram iniciativas para complementação dos índices, levando em conta os impactos antrópicos. São exemplos deste propósito o ISTO Índice de Substâncias Tóxicas e Organolépticas (CETESB, 2017), voltado às águas 
superficiais, o SEQ ESO Système d’Évaluation de la Qualité - Eaux Souterraines (CADILHAC E ALBINET, 2003) e o IQASB (ALMEIDA, 2007), direcionados ao compartimento subterrâneo.

Várias metodologias, principalmente da ciência estatística, foram incorporadas e se consolidaram como ferramentas auxiliares para caracterização da qualidade da água. No trabalho desenvolvido por Ferreira et al. (2015) foram utilizadas técnicas de Análise de Componentes Principais em uma adaptação do Water Quality Index National Sanitation Foundation (BROWN ET AL., 1970). Cecconello, Centeno e Guedes (2018) utilizaram a análise multivariada para propor um índice de qualidade de água modificado (IQAMOD) a partir do Índice de Qualidade de Água - IQA (CETESB, 2017).

O uso de critérios estatísticos reduz os pressupostos subjetivos da formulação dos índices, entretanto são procedimentos complexos e nem sempre adaptáveis aos dados disponíveis. Assim, os IQA's permanecem como recursos fundamentais para dar conhecimento sobre a qualidade dos compartimentos hídricos.

Para compor um IQA, as concentrações das substâncias (parâmetros) em uma amostra de água são avaliadas, e as notas de qualidade são atribuídas de acordo com os teores encontrados. Em seguida, os parâmetros são juntados por meio das chamadas estruturas de agregação, que podem ser: estruturas de agregação aditivas e multiplicativas ou métodos exclusivos.

As formas de agregação aditivas e multiplicativas dos parâmetros, em geral produzem as chamadas "zonas de ambiguidade" (quando todos os subíndices indicam a qualidade da água aceitável, mas o índice agregado mostra que não) ou eclipse (quando a contagem do índice "esconde" o teor inaceitável de um ou mais parâmetros do índice). Ademais, esses índices impedem a inclusão de novos parâmetros, sendo inflexíveis (parâmetros determinados) e válidos para determinada área e, portanto de uso limitado (ALMEIDA, 2012).

Os métodos exclusivos, como o próprio nome anuncia, baseiam-se na escolha de apenas um dos valores do universo considerado, o qual será o representante na composição final do índice, em detrimento dos demais valores. Dentre estes, os mais usuais são os operadores máximo e mínimo. Como indicado no próprio nome: o operador máximo recupera o maior valor em um grupo de dados, enquanto o operador mínimo resgata o menor valor. Ou seja, a nota final do índice poderá ser a menor nota 
dentre todas as substâncias analisadas (operador mínimo) ou a maior delas (operador máximo) (ALMEIDA, 2012).

\subsubsection{A formulação do e-IQUAS}

Uma das premissas do e-IQUAS é que todas as substâncias presentes em uma amostra de água podem ser analisadas, entretanto algumas delas são mais proeminentes e produzem efeitos na qualidade do recurso. Neste sentido, os parâmetros foram reunidos em sete "grupos de alterações", que associam os efeitos da sua presença: Agrotóxicos e Pesticidas, Elementos Filtráveis e Partículas, Matéria Orgânica e Nutriente, Micro-organismos, Micropoluentes Minerais, Micropoluentes Orgânicos, Mineralização e Salinidade.

Para cálculo do índice, primeiramente são atribuídas notas a cada parâmetro analisado. Para isso, o teor encontrado na amostra é confrontado com faixas de concentração associadas à qualidade requerida, em uma escala de que varia de 0 (ruim) a 100 (ótima qualidade). O Quadro 1 exemplifica a metodologia mostrando as faixas de teores e as categorias aplicáveis ao parâmetro Turbidez. Ou seja, exemplificando: caso uma amostra apresente um teor de turbidez de 4,6 UT, será atribuída a nota 60 ao parâmetro Turbidez.

Quadro 1 - Concentrações X Nota do parâmetro - Turbidez

\begin{tabular}{|c|c|c|c|c|}
\hline Parâmetro: Turbidez & \multicolumn{2}{|c|}{$\begin{array}{c}\text { Faixas de Teores } \\
\text { (UT) }\end{array}$} & Nota & Conceito \\
\hline Concentração abaixo de um limite guia & $\mathrm{N}=0$ & $\mathrm{~N}=<4,0$ & 80 & Ótima \\
\hline $\begin{array}{c}\text { Concentração entre dois valores de } \\
\text { relevância }\end{array}$ & $\mathrm{N}>4,0$ & $\mathrm{~N}=5,0$ & 60 & Aceitável \\
\hline $\begin{array}{c}\text { Concentração entre outros dois valores de } \\
\text { relevância }\end{array}$ & $\mathrm{N}>5,0$ & $\mathrm{~N}=<15,0$ & 40 & $\begin{array}{c}\text { Não } \\
\text { potável }\end{array}$ \\
\hline $\begin{array}{c}\text { Concentração acima do admissível } \\
\text { Inapta }\end{array}$ \\
\hline
\end{tabular}

Fonte: ALMEIDA (2012)

Em seguida, aplica-se o método de agregação do "valor mínimo" para conferir a nota do "grupo de alteração" (ou subíndice). Cada subíndice agrupa uma gama de parâmetros que produzem o mesmo efeito sobre a qualidade da água, quando 
presentes em uma amostra. Ainda usando como exemplo a Turbidez, esta substância juntamente com a Cor, Ferro e Manganês formam o grupo dos "Elementos Filtráveis e Partículas". Ou seja, caso em uma amostra os teores de Cor, Ferro e Manganês obtivessem a nota 80 e a Turbidez auferisse a nota 60 , então a nota do grupo de alteração "Elementos Filtráveis e Partículas" seria 60.

O método do "valor mínimo" é considerado o mais adequado aos propósitos de um índice flexível, como o e-IQUAS. Esta estrutura mostra-se apropriada, especialmente quanto a não restrição ao número de parâmetros, como também quanto à simplicidade dos cálculos. Este método de agregação foi escolhido para o cálculo dos subíndices dos grupos de alteração e do índice final propostos neste trabalho.

Portanto, ao Índice de Qualidade de Uso da Água Subterrânea (e-IQUAS) é atribuída a menor nota entre todas as notas dos grupos de alterações que fazem parte do índice, na escala mostrada no Quadro 2.

Quadro 2 - Escala de notas do e-IQUAS

\begin{tabular}{|c|c|c|}
\hline e-IQUAS & Qualificação & Semáforo \\
\hline 80 & Otima & \\
\hline 60 & Boa & \\
\hline 40 & Regular & \\
\hline 20 & Ruim & \\
\hline
\end{tabular}

\section{MATERIAIS E MÉTODOS}

O projeto, desenvolvimento e verificação de um aplicativo exigem: definição das regras de negócio e funcionalidades atendidas, e escolha das ferramentas computacionais e do conjunto de dados utilizados. No caso em tela, acrescenta-se a condição de que fossem utilizados recursos da filosofia de software livre. Esta seção é dedicada a apresentar a metodologia de desenvolvimento do aplicativo e-IQUAS.

\subsection{Arquitetura do sistema}

As estruturas estática e comportamental do sistema foram modeladas usando os diagramas de Linguagem de Modelagem Unificada (UML, do inglês Unified Modeling Language), por meio de "Diagramas de Classe" e "Diagramas de Casos de Usos". A UML, surgida nos anos noventa do século passado, de acordo com Rumbaugh (2004) é uma linguagem de propósito geral de modelagem visual que é 
usada para especificar, visualizar, construir e documentar os artefatos de um sistema ou aplicativo.

As classes são as representações de objetos do mundo real, com atributos, comportamentos e relacionamentos com outros objetos. Uma amostra de água e os parâmetros medidos na amostra são exemplos de classes. Os relacionamentos são associações entre as classes, como, por exemplo: uma amostra contém vários parâmetros. O diagrama de classes do sistema e-IQUAS está representado na Figura 1.

Os "diagramas dos casos de uso" e as "descrições dos casos de uso" complementam a modelagem. Estes artefatos representam a estrutura comportamental do sistema. Os procedimentos para "Cálculo do e-IQUAS", reproduzidos na Figura 2, mostram os processos para determinação do índice, depois da inclusão dos dados da amostra.

Figura 1 - Diagrama de classes do sistema e-IQUAS

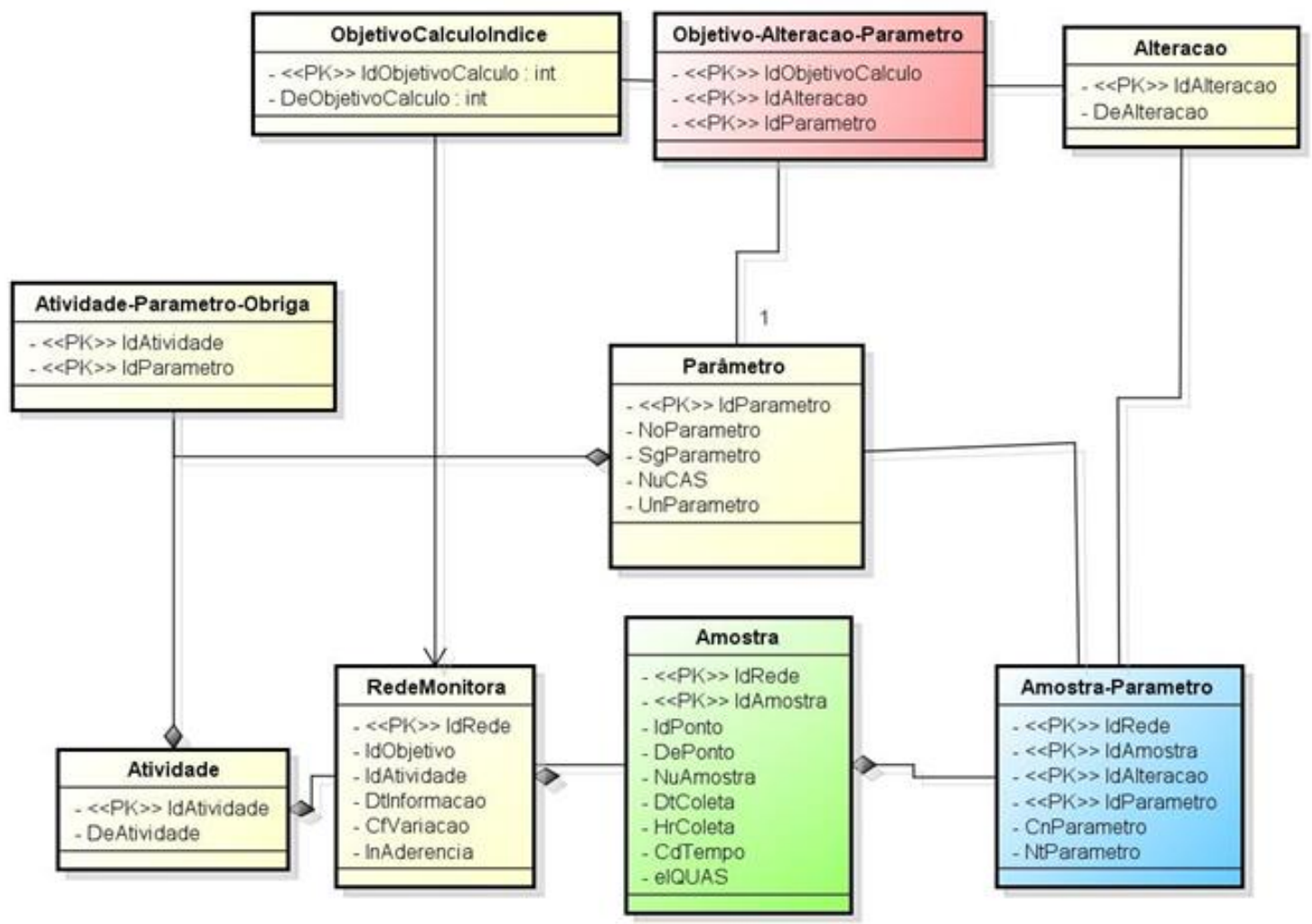

Fonte: Almeida (2012) 
Figura 2 - Diagrama do caso de uso "Cálculo do e-IQUAS"

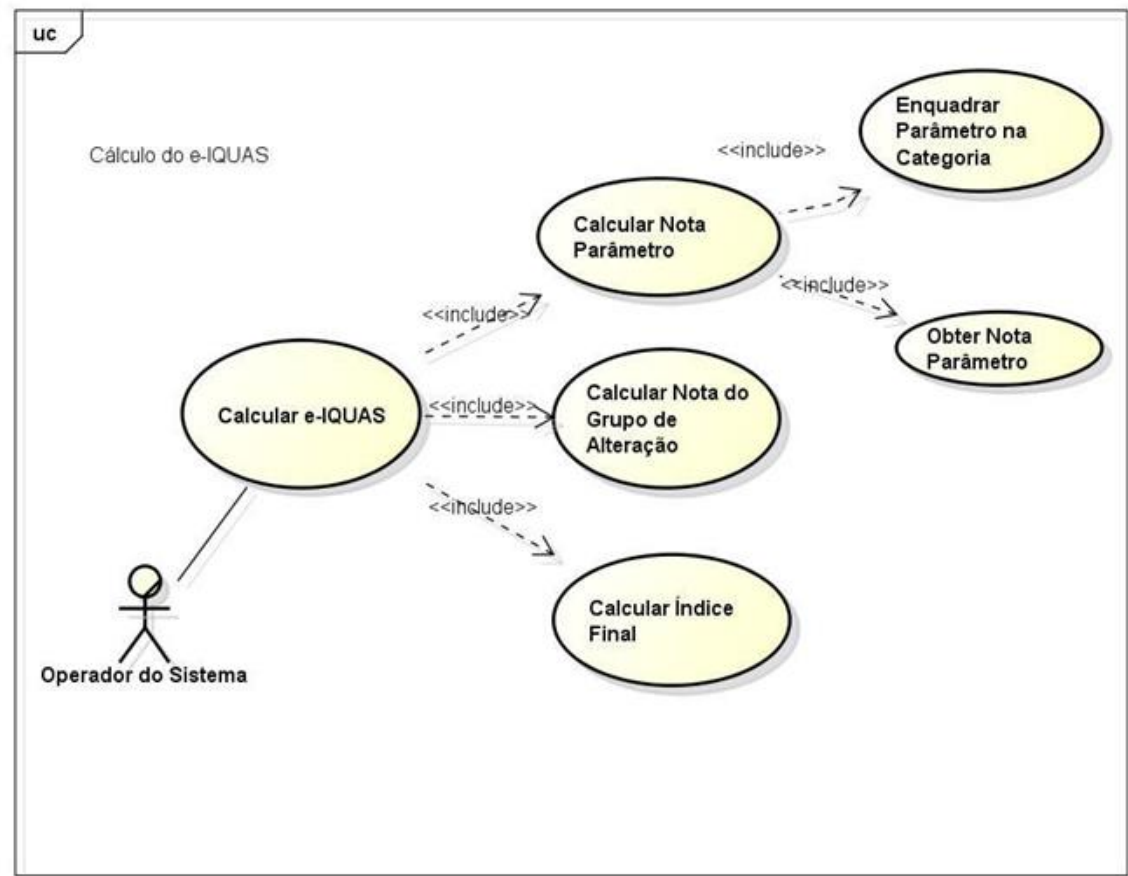

Fonte: Almeida (2012)

\subsection{Projeto do Banco de Dados}

Um banco de dados consiste de múltiplas relações que armazenam de forma estruturada as informações sobre determinado negócio, de modo que não haja repetição de dados e armazenamento de valores nulos. De acordo com Silberschatz, Korth e Sudarshan (2010), os sistemas de banco de dados são um grupo de ferramentas para descrever os dados, as relações entre eles, a semântica e as restrições dos relacionamentos.

Para manipular dados usando um sistema gerenciador de banco de dados (SGBD) é preciso que as classes de dados da modelagem UML sejam representadas na forma de tabelas físicas e que todas as relações estabelecidas entre elas sejam representadas.

A representação por meio de um diagrama de entidade relacionamento (DER) permite que sejam reproduzidos fisicamente no banco de dados os relacionamentos previstos na modelagem lógica, como por exemplo, nas definições do aplicativo eIQUAS, a exigência de que "um grupo de alterações contenha um ou vários parâmetros" e que este mesmo "parâmetro só possa estar relacionado a um, e apenas um, grupo de alterações". 
O diagrama de entidade relacionamento (Entity Relationship Diagram - ERD), que descreve o modelo de dados do sistema e-IQUAS, foi elaborado na ferramenta visual MySQL Workbench (2012), e é apresentado na Figura 3 - Diagrama Entidade Relacionamento (DER) do Sistema e-IQUAS.

A partir do diagrama foram utilizadas as linguagens de definição de dados e de consulta estruturada, respectivamente Data Definition Language (DDL) e Structured Query Language (SQL), para criação das tabelas no ambiente de desenvolvimento do MySQL Community Server (MYSQL, 2011). Estes procedimentos resultaram na criação de onze tabelas, que integralizam o modelo de dados do aplicativo e-IQUAS.

\subsection{Linguagens e plataformas de desenvolvimento}

A plataforma de desenvolvimento escolhida para criar o aplicativo faz parte do conjunto de ferramentas de software livre. De acordo com a Free Software Foundation (FSF, 2020) um software é livre se oferece ao usuário a liberdade de compartilhá-lo, estudá-lo e modificá-lo. A organização esclarece ainda que "Software livre" não significa "não comercial", ou seja, o software livre pode ter uso, desenvolvimento e distribuição comercial, mas o usuário mantém as liberdades de executar, copiar, distribuir, estudar, mudar e melhorar o software (FSF, 2020). Foram selecionadas linguagens de programação, seus frameworks e bibliotecas e o sistema gerenciador de banco de dados (SGBD).

Decidiu-se utilizar no back-end a linguagem PHP 7.2, com recursos Laravel (V5.4). O Laravel é uma estrutura de aplicativos (framework), livre e de código aberto, para o desenvolvimento de aplicações em PHP, que facilita a programação de tarefas comuns usadas na maioria dos projetos da web (LARAVEL, 2020).

No front-end utilizou-se as linguagens de programação HTML5 e JavaScript, com recursos do jQuery. O jQuery é produto de um dos projetos da OpenJS Foundation, organização de apoio ao desenvolvimento colaborativo de JavaScript e tecnologias da web. Trata-se de uma biblioteca de funções JavaScript que simplifica o processo de passagem e manipulação de documentos HTML (JQUERY, 2020).

$\mathrm{Na}$ definição da aparência dos elementos gráficos das páginas (cores, fontes, espaçamento) foi utilizado CSS 3 (Cascading Style Sheets) com a biblioteca Bootstrap 4. Desenvolvido inicialmente para o microblog Twiter, o Bootstrap é uma ferramenta gratuita, com código-fonte aberto, para desenvolvimento de projetos responsivos para 
dispositivos móveis na web, com componentes pré-construídos e plugins com jQuery (BOOTSTRAP, 2020).

Figura 3 - Diagrama Entidade Relacionamento (DER) do Sistema e-IQUAS e-IOUAS

Diagrama Entidade - Relacionamento do Sistem a e-IQUAS

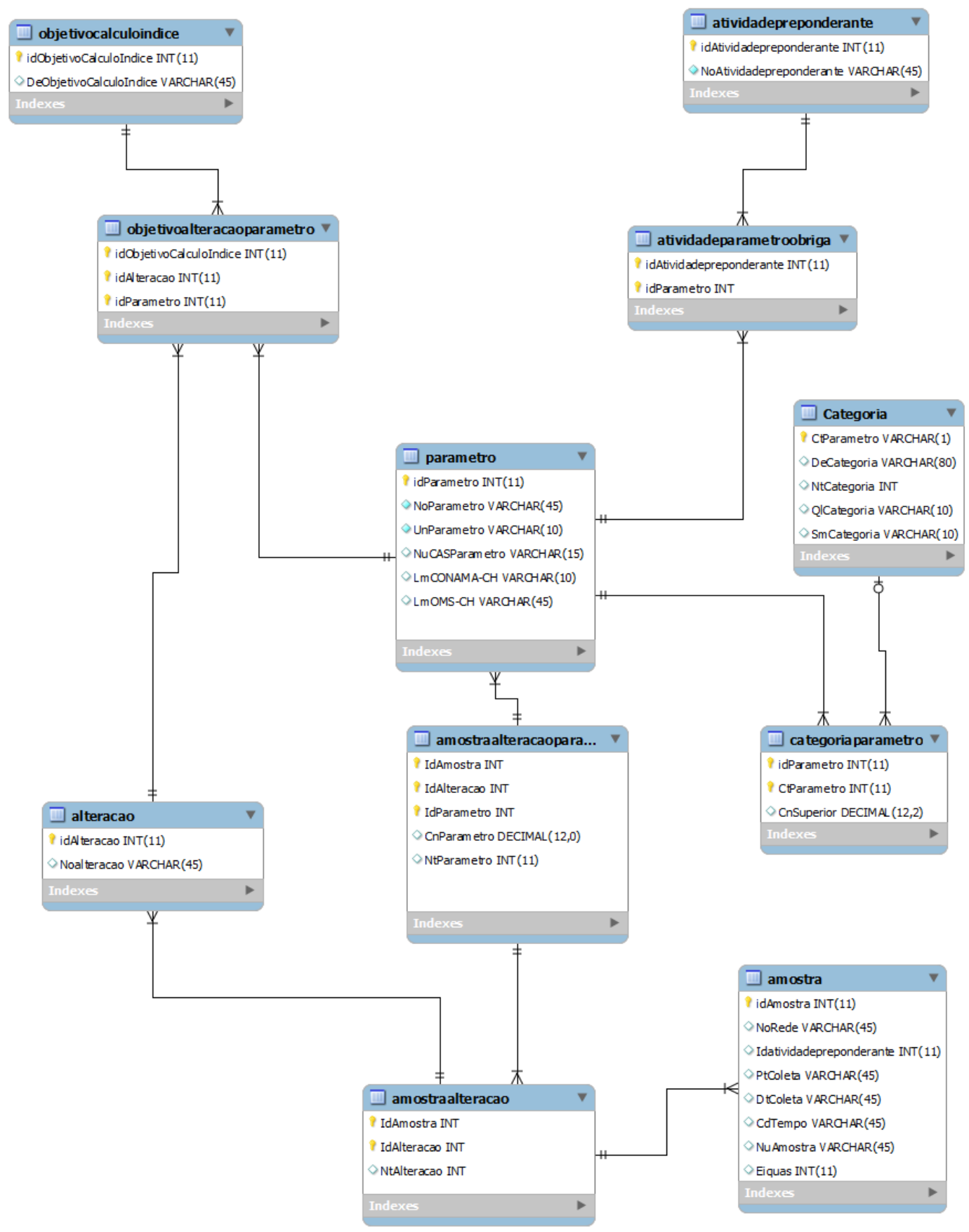

Fonte: Almeida (2012) 
Para armazenamento dos dados a escolha foi o SGBD MySQL, versão 4.1, também do conjunto de ferramentas livres, com acesso aos dados por meio da linguagem de pesquisa SQL (Structured Query Language). Ele pode ser executado em praticamente todas as plataformas e sistemas operacionais, é um banco de dados maduro, robusto e fácil de trabalhar (WALKER, 2017).

\subsection{Funcionalidade e interface gráfica}

O cadastro de parâmetros e dos grupos de alterações é obrigatório para utilização do sistema e-IQUAS. Eles armazenam os dados básicos sobre as substâncias que podem ser analisadas para caracterizar a qualidade de uma amostra de água, como: nome, unidade de medida, limites de concentração aceitos por instituições de controle, tipo de alteração que a presença da substância promove na qualidade da água, dentre outros. Estes dados são previamente cadastrados e salvos na base de dados. Na Figura 4 e 5 estão os formulários para cadastramento dos parâmetros. Em todos eles, botões interrogativos permitem que o usuário obtenha informações sobre o conteúdo a ser digitado.

Figura 4 - Cadastramento de Parâmetro - Dados Básicos

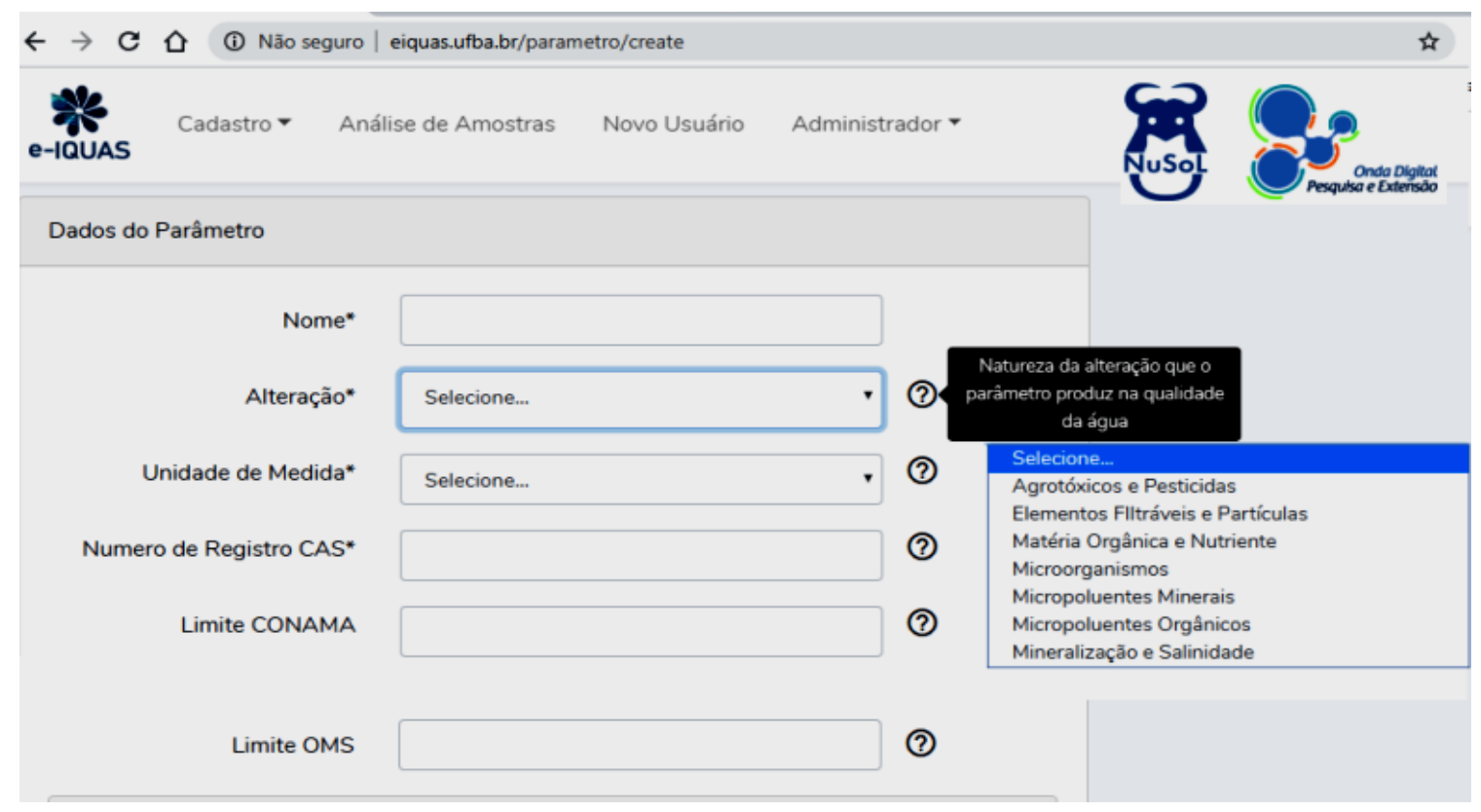


Figura 5 - Cadastramento de Parâmetro - Categorias de Qualidade

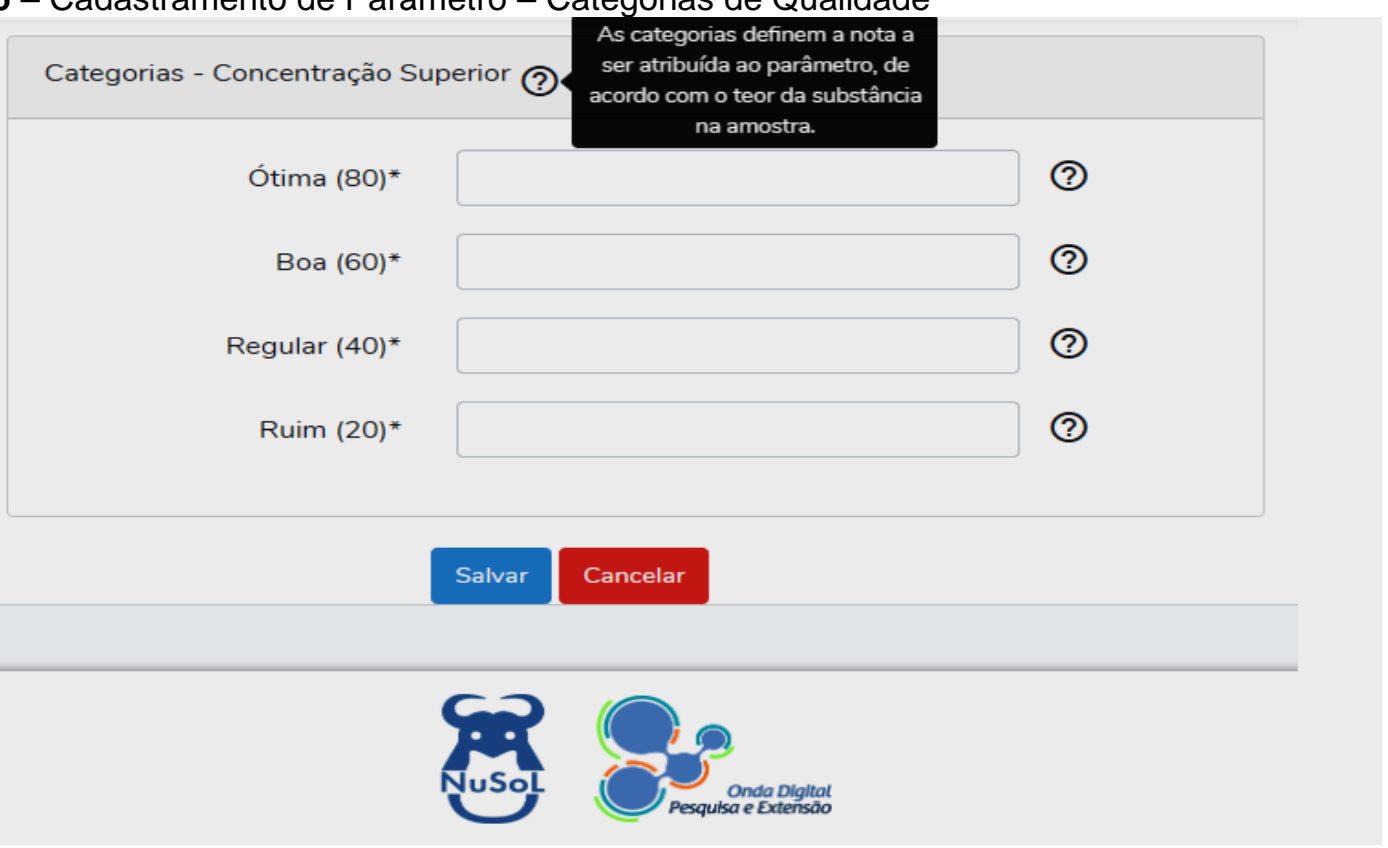

O cálculo do índice de qualidade de água de uma amostra se dá pela inclusão dos resultados das medidas de concentração dos parâmetros amostrados e execução da funcionalidade "Calcular e Exibir". Nas Figuras 6 e 7 estão representados o formulário de recepção dos dados gerais de uma amostra e inserção dos resultados dos parâmetros, respectivamente.

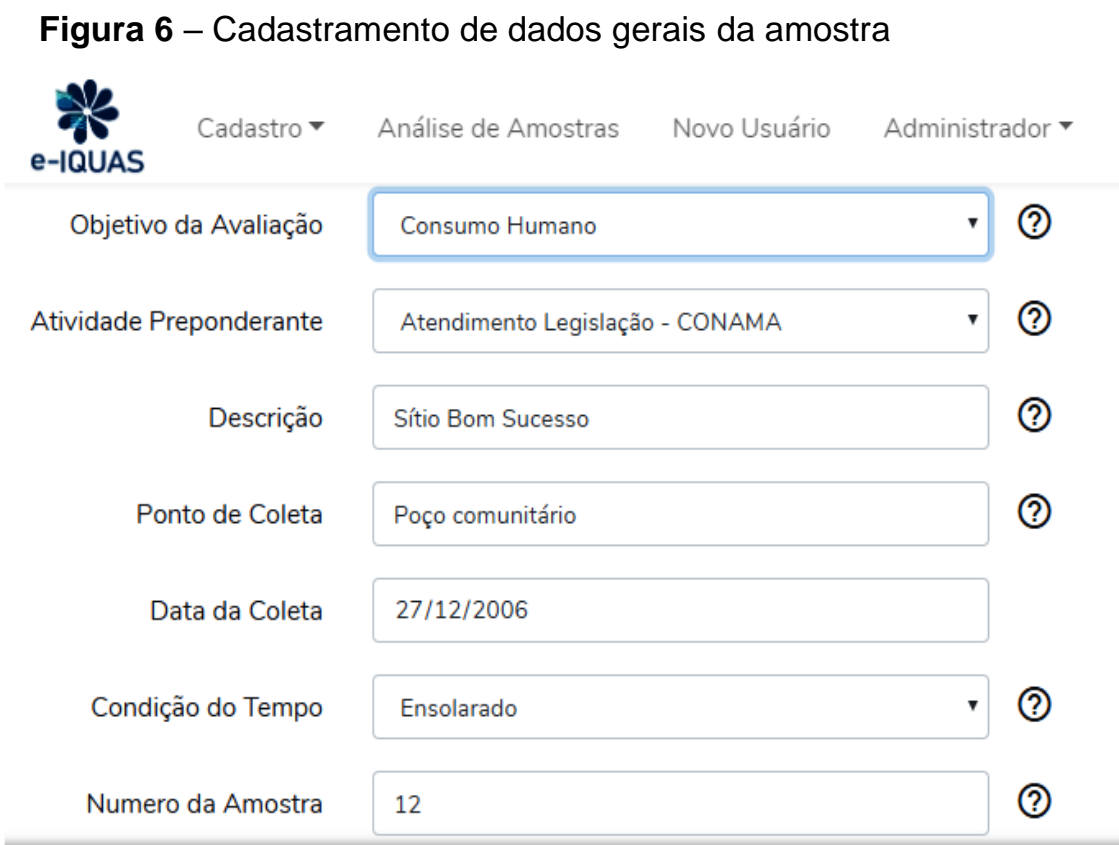




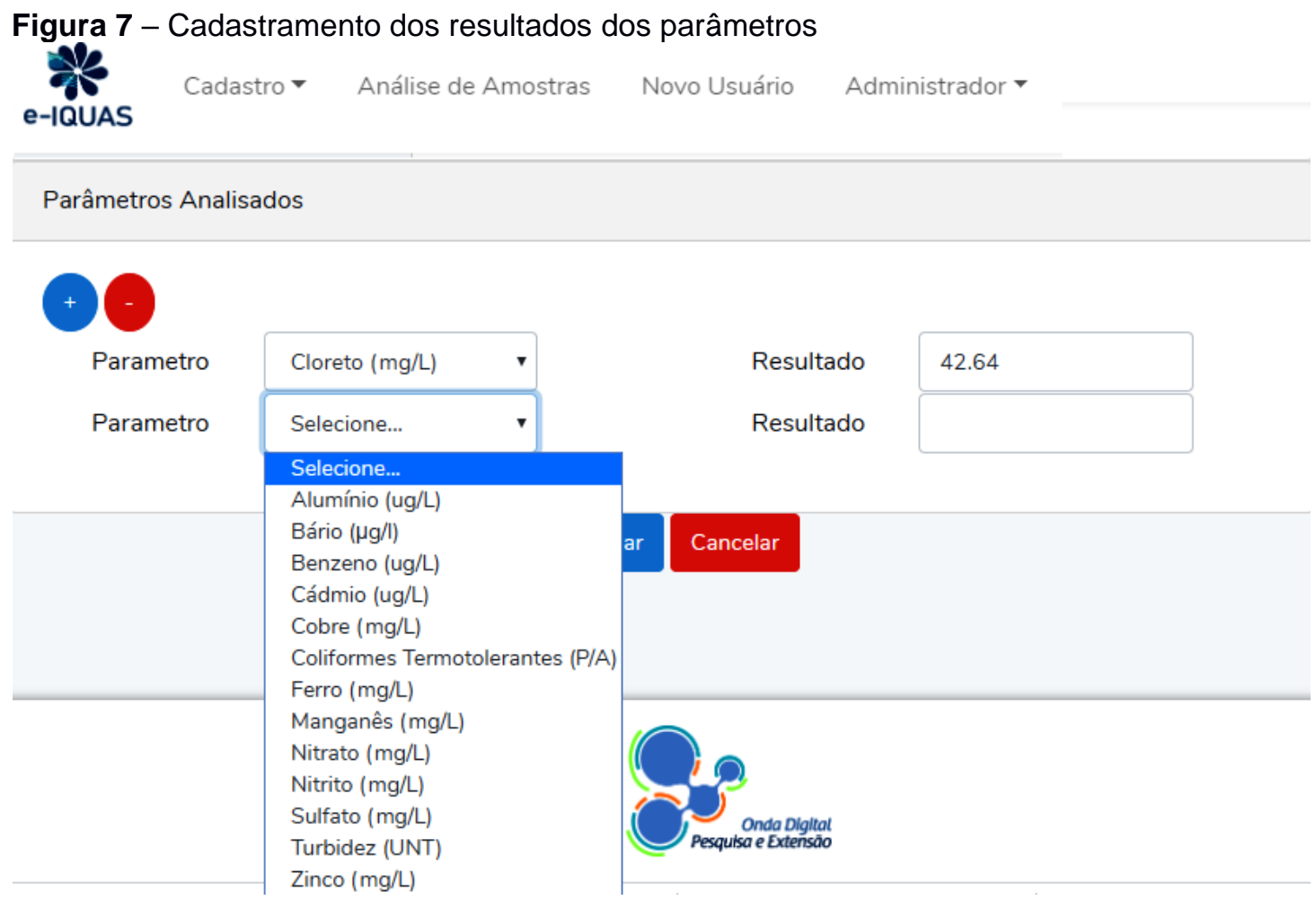

Ao concluir a digitação dos resultados dos parâmetros o usuário comanda o cálculo, o sistema verifica as concentrações e compara com os padrões estabelecidos previamente. Em seguida exibe o resultado do cálculo do índice de qualidade eIQUAS.

\subsection{Acesso ao aplicativo e-IQUAS}

O aplicativo e-IQUAS foi desenvolvido no âmbito do Núcleo de Software Livre (NUSOL) do Grupo de Pesquisa e Extensão em Informática, Educação e Sociedade Onda Digital da Universidade Federal da Bahia, e foi disponibilizado na rede mundial de computadores com apoio institucional da superintendência de tecnologia da informação (STI) da mesma universidade.

O aplicativo pode ser acessado diretamente no seu endereço web (www.eiquas.ufba.br). O acesso também pode ser feito por meio da plataforma colaborativa ACQUAINDEX criada para incentivar a difusão de informações sobre a qualidade da água, no ambiente acadêmico, e, sobretudo para a sociedade em geral.

A Figura 8 reproduz a tela de entrada no aplicativo, acessado a partir de dispositivo móvel. 
Figura 8 - Tela de entrada do sistema e-IQUAS

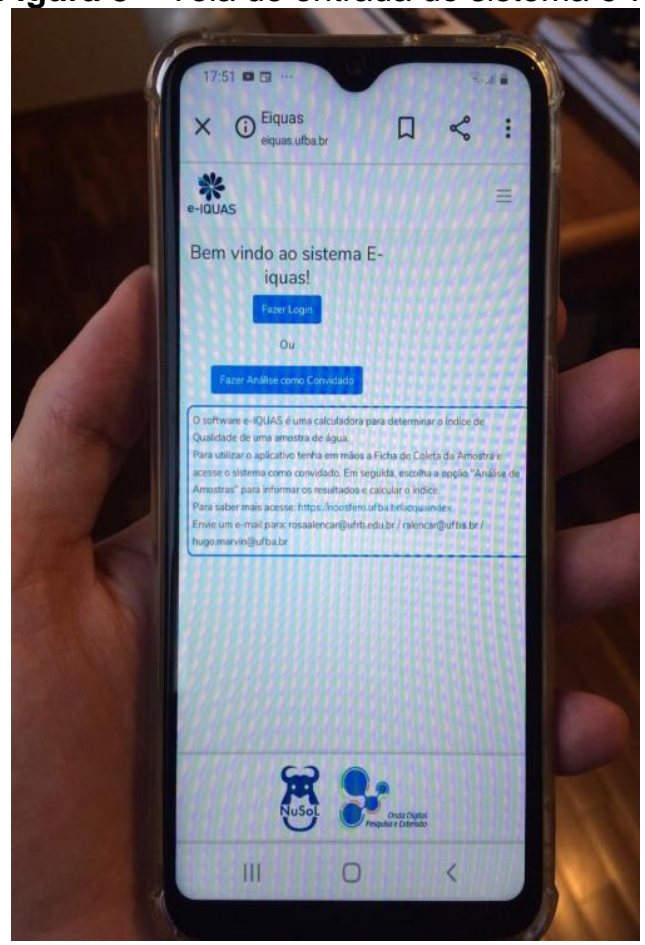

\section{RESULTADOS E DISCUSSÃO}

\subsection{Estudo de caso - Amostra de Água}

Para realização dos testes do aplicativo e-IQUAS foram utilizados dados secundários disponibilizados em sites institucionais de órgãos ambientais. Neste trabalho são demonstrados os resultados obtidos a partir das Séries Históricas de Monitoramentos da Qualidade das Águas Superficiais no Estado de Minas Gerais, referentes ao período de 2012 a 2016, disponíveis no repositório institucional do Instituto Mineiro de Gestão das Águas (2020) com acesso pelo identificador: http://200.198.57.118:8080/jspui/handle/123456789/414.

As amostras foram coletadas na Unidade de Planejamento e Gestão de Recursos Hídricos (UPGRH) JQ3 - Médio / Baixo Rio Jequitinhonha. O e-IQUAS foi calculado, e os resultados foram comparados com o mapa panorama de qualidade das águas superficiais das Bacias Hidrográficas do Médio e Baixo Rio Jequitinhonha, disponível no repositório institucional do Instituto Mineiro de Gestão das Águas (2016), com acesso pelo identificador http://200.198.57.118:8080/jspui/handle/123456789/1964. 
Quatro das amostras da série histórica da UPGRH JQ3 - Médio / Baixo Rio Jequitinhonha - Estação JE21 estão relacionadas no Quadro 3. Tem-se as descrições dos pontos, datas da coleta, teores dos parâmetros medidos e resultados do e-IQUAS. O cálculo do índice foi realizado diretamente no site www.eiquas.ufba.br.

Quadro 3 - Amostragens na UPGRH JQ3 - Médio / Baixo Rio Jequitinhonha

\begin{tabular}{|c|c|c|c|c|}
\hline \multirow{3}{*}{$\begin{array}{c}\text { Ponto de Coleta } \\
\text { Descrição }\end{array}$} & \multirow{2}{*}{\multicolumn{2}{|c|}{$\begin{array}{c}\text { Estação JE021 } \\
\text { Rio Jequitinhonha na cidade de } \\
\text { Jequitinhonha }\end{array}$}} & \multirow{2}{*}{\multicolumn{2}{|c|}{$\begin{array}{c}\text { Estação JE028 } \\
\text { Rio Jequitinhonha a Jusante de } \\
\text { Itaobim }\end{array}$}} \\
\hline & & & & \\
\hline & 03/02/2016 & $03 / 08 / 2016$ & 02/02/2016 & $02 / 08 / 2016$ \\
\hline Amostra & $\begin{array}{c}\text { JE021 - 1TR - } \\
\text { AM01 } \\
\end{array}$ & JE021 - 3TR - AM02 & $\begin{array}{c}\text { JE028 - 1TR - } \\
\text { AM01 } \\
\end{array}$ & JE028 - 3TR - AM02 \\
\hline $\begin{array}{l}\text { Condição de } \\
\text { tempo }\end{array}$ & Bom & Chuvoso & Bom & Nublado \\
\hline Escherichia Coli & 479,8 & 132,3 & 775,7 & 573,1 \\
\hline Fósforo $(\mathrm{P})$ & 0,13 & $<0,02$ & 0,19 & $<0,02$ \\
\hline DBO & $<2$ & $<2$ & $<2$ & $<2$ \\
\hline Nitrato $\left(\mathrm{NO}_{3}{ }^{-}\right)$ & 0,58 & 0,59 & 0,5 & 0,79 \\
\hline $\mathrm{NH}_{3} \mathrm{~T}$ & 0,29 & $<0,1$ & 0,4 & $<0,1$ \\
\hline Arsênio & $<0,001$ & $<0,001$ & $<0,001$ & $<0,001$ \\
\hline Cianetos (CN-) & 0,002 & $<0,002$ & $<0,002$ & $<0,002$ \\
\hline Chumbo (Pb) & 0,00994 & $<0,005$ & 0,018 & $<0,005$ \\
\hline Cobre ( $\left.\mathrm{Cu}_{\text {diss }}\right)$ & $<0,004$ & $<0,004$ & $<0,004$ & $<0,004$ \\
\hline Zinco $(Z n)$ & 0,0261 & 0,0616 & 0,0392 & 0,0226 \\
\hline Cromo (Cr) & $<0,04$ & $<0,04$ & $<0,04$ & $<0,04$ \\
\hline Cádmio (Cd) & $<0,0005$ & $<0,0005$ & $<0,0005$ & $<0,0005$ \\
\hline Fenóis Totais & $<0,002$ & $<0,002$ & $<0,002$ & $<0,002$ \\
\hline Mercúrio $(\mathrm{Hg})$ & $<0,2$ & $<0,2$ & $<0,2$ & $<0,2$ \\
\hline e-IQUAS & 60 (Aceitável) & 80 (Ótima) & 20 (Inapta) & 80 (Ótima) \\
\hline
\end{tabular}

Fonte: Instituto Mineiro de Gestão das Águas (2020)

Os critérios para seleção das amostras foram baseados na disponibilidade de parâmetros que permitissem caracterizar minimamente uma massa de água. Esse cuidado é necessário para dar robustez e credibilidade aos resultados encontrados.

\subsection{Teste do aplicativo}

Os parâmetros cujos resultados estavam abaixo do limite de detecção, ou seja, aqueles cujo resultado é precedido pelo sinal "menor do que" (<), foram descartados. Os demais resultados foram digitados, conferidos e a operação foi confirmada. Em resposta, o aplicativo disparou o cálculo do e-IQUAS. Os quadros 4 e 5 mostram a 
sequência das operações para inclusão da amostra JE021 - 1TR - AM01. E no quadro 6 tem-se o relatório completo do e-IQUAS.

Quadro 4 - Inserção dos dados da amostra JE021 - 1TR - AM01

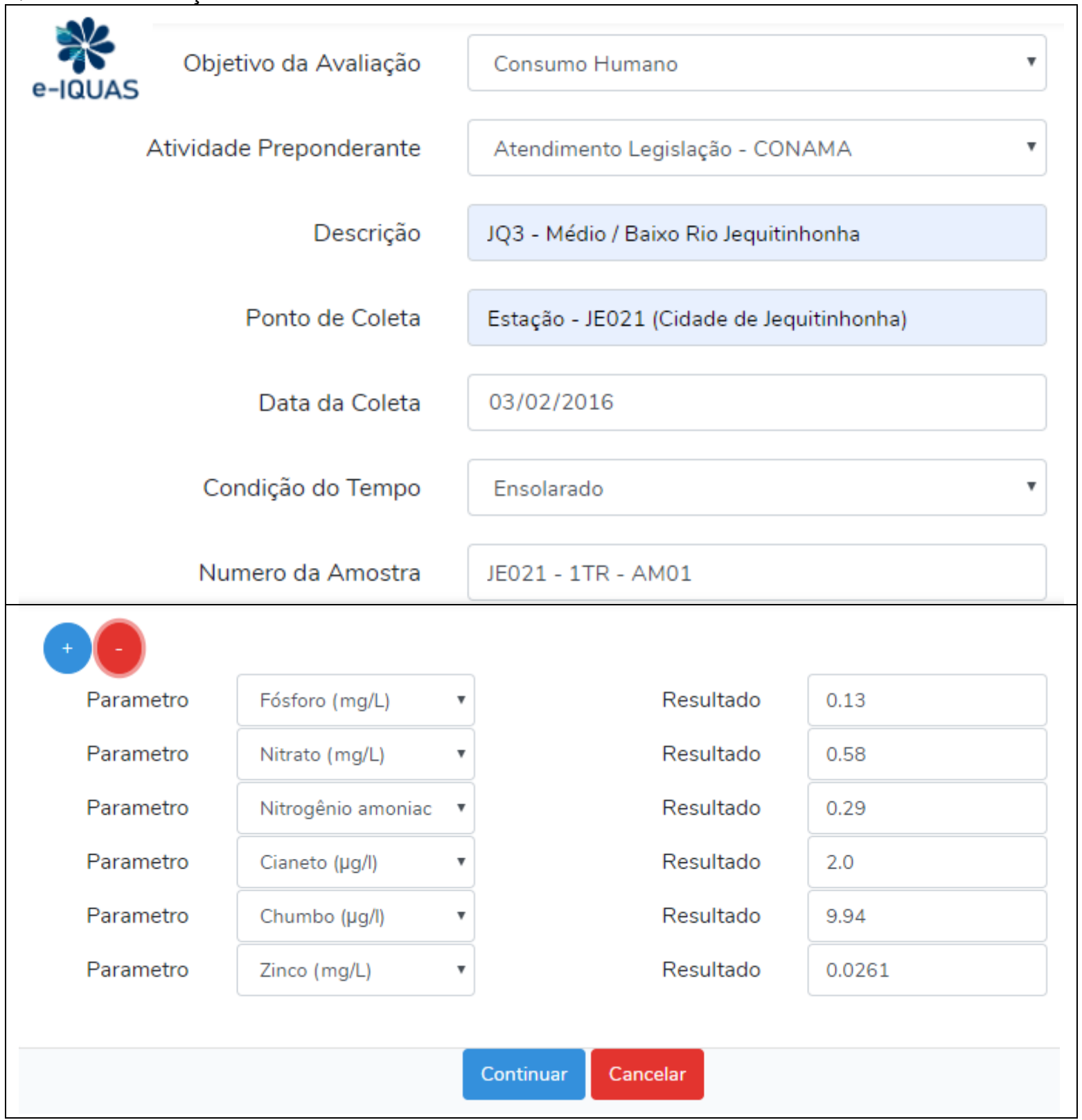


Quadro 5 - Confirmação dos dados da amostra JE021 - 1TR - AM01

Dados da Amostra

\begin{tabular}{|c|c|}
\hline Dbjetíro: & Consumo Humano \\
\hline Atividade Preponderanter & Atendimento Legislação - CONAMA \\
\hline Descriçăax: & JQ3 - Médio / Baixo Rio Jequitinhonha \\
\hline Ponto de Coletex & Estação - JE021 (Cidade de Jequitinhonha) \\
\hline Data da Coletax & $03 / 02 / 2016$ \\
\hline Condição do Tempx & Chuvoso \\
\hline Nuimero da Amostra & JEO21 - 1TR - AMO1 \\
\hline
\end{tabular}

Parâmetros:

\begin{tabular}{|l|l|l|l|}
\hline Parametro: & Fósforo $(\mathrm{mg} / \mathrm{L})$ & Resultadx: & 0.13 \\
\hline Parametro: & Nitrogênio amoniacal total $(\mathrm{mg} / \mathrm{l})$ & Resultado: & 0.29 \\
\hline Parametro: & Nitrato $(\mathrm{mg} / \mathrm{L})$ & Resultado: & 0.58 \\
\hline Parametro: & Chumbo $(\mu \mathrm{g} /)$ & Resultadx & 9.94 \\
\hline Parametro: & Cianeto $(\mu \mathrm{g} /)$ & Resultado: & 2.00 \\
\hline & \multicolumn{1}{|r}{ Confirmar } & Cancelar \\
\hline
\end{tabular}

Quadro 6 - e-IQUAS da amostra JE021 - 1TR - AM01 Indice de Qualidade da Água (E-IQUAS)

\begin{tabular}{|c|c|}
\hline Amostra IE021 - 1TR - AM01 & DatadaColatar 03/02/2016 \\
\hline Pontode Coleta Estaça - jEO21 (Civlade de lequitinboeiha) & Condigäo do Tentpor Chuvoso \\
\hline \multicolumn{2}{|l|}{ Atrividade Pheponderante: Atend imento Legislaçāo - CONAMA } \\
\hline nescripisio JQ3 - Medio / Baixo Rio Jequitinhonha & \\
\hline
\end{tabular}

Grupo Modificador de qualidade:

\begin{tabular}{|c|c|}
\hline Materia Ningtrica e Whitionte & 60 \\
\hline Nitrato: & 80 \\
\hline Fósforo: & 60 \\
\hline Nitroginio ameniacal totat: & 80 \\
\hline Monopolusntes Mneras: & 60 \\
\hline Chumbe: & 60 \\
\hline Clanetex & 80 \\
\hline
\end{tabular}

Resultado:

\begin{tabular}{|c|c|}
\hline 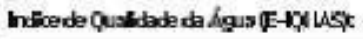 & 60 \\
\hline Indiogqua a Amosita Esth & Boa \\
\hline
\end{tabular}




\subsection{Avaliação do experimento}

O e-IQUAS das quatro amostras foram confrontados com o panorama de qualidade das águas superficiais das Bacias Hidrográficas do Médio e Baixo Rio Jequitinhonha no primeiro trimestre de 2016 (INSTITUTO MINEIRO DE GESTÃO DAS ÁGUAS, 2016). Os resultados demonstram a concordância entre os índices calculados e o quadro de parâmetros indicativos registrados no mapa.

De acordo com o documento, no primeiro trimestre de 2016, as Estações JE021 e Estação JE028 apresentaram não conformidade para os parâmetros indicadores de enriquecimento orgânico. A Estação JE028 também estava não conforme para os parâmetros relacionados à presença de substâncias tóxicas. A nota do e-IQUAS para a Estação JE021 indica uma água de qualidade aceitável (e-IQUAS $=60)$, refletindo a não conformidade do parâmetro Fósforo (Nota do Parâmetro $=60$ ), indicador de enriquecimento orgânico. A nota também espelha a presença do Chumbo (Nota do Parâmetro $=60$ ), em concentração acima do valor máximo tolerado para água de ótima qualidade. Já a nota do e-IQUAS para a Estação JE028, da mesma maneira reflete a presença dos parâmetros já mencionados, alcançando a caracterização de água de qualidade inapta (e-IQUAS $=20$ ).

Para consolidar a verificação do desempenho do aplicativo e-IQUAS foi realizada uma massa de testes com resultados fictícios e também amostras reais, colhidas em sites institucionais (Quadro 7). Os resultados indicam que o aplicativo eIQUAS funciona adequadamente e pode ser amplamente utilizado pelo público em geral. As janelas de ajuda (?) facilitam sobremaneira a digitação dos campos de descrição livre, e os campos padronizados estão em acordo com as premissas de operação do cálculo do índice. 
Quadro 7 - Lista de Amostras de testes do aplicativo e-IQUAS

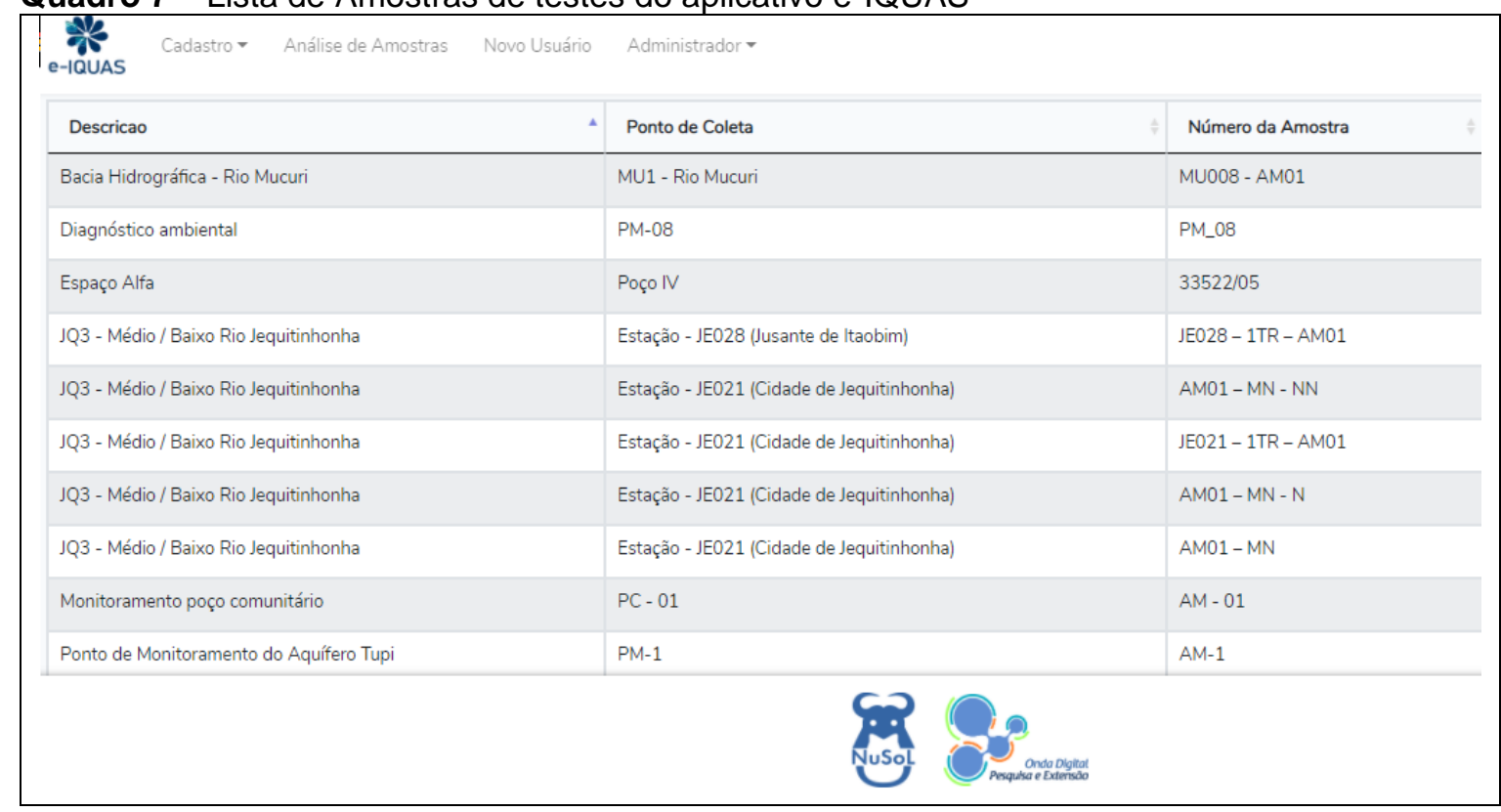

\subsection{Acesso ao aplicativo}

O aplicativo e-IQUAS foi modelado para calcular e exibir de forma objetiva e simples um índice de qualidade de água flexível na seleção de parâmetros indicadores, sejam eles físico, químicos ou biológicos. Os módulos são interligados e permitem o cumprimento de todas as funcionalidades de forma integrada.

O e-IQUAS pode ser acessado diretamente no site (www.eiquas.ufba.br) ou por meio da plataforma colaborativa ACQUAINDEX (https://noosfero.ufba.br/acquaindex).

\section{CONCLUSÃO E RECOMENDAÇÕES}

O desenvolvimento do aplicativo e-IQUAS livre trouxe aprendizados importantes. Em primeiro lugar confirma o entendimento de que: o uso de ferramentas da filosofia do software livre é uma alternativa importante para democratização do acesso à informação, na medida em que possibilita que um programa seja executado, copiado, modificado e redistribuído irrestritamente. Além disso, atesta que: a simplicidade e flexibilidade do modelo do e-IQUAS na seleção, inclusão e supressão de parâmetros, é uma característica relevante e aumenta as possibilidades de uso do aplicativo. 
Ademais, como o e-IQUAS foi projetado com a preocupação de que venha a ser utilizado de forma ampla, por esta razão, são bem vindas as iniciativas para aprimorá-lo.

Julga-se como oportunas pesquisas para programação de funcionalidades para determinação da localização geográfica do ponto de coleta; a entrada de dados em lote para campanhas de monitoramento e o armazenamento de amostras em banco de dados. Elas proporcionarão o acompanhamento da evolução temporal da qualidade da água em determinada localização. Como também incorporam novas possibilidades ao monitoramento dos mananciais.

\section{AGRADECIMENTOS}

Aos pesquisadores e colaboradores do Núcleo de Software Livre (NUSOL) do Grupo de Pesquisa e Extensão em Informática, Educação e Sociedade - Onda Digital da Universidade Federal da Bahia e da Superintendência de Tecnologia da Informação (STI) da mesma universidade, pelo apoio, colaboração e estrutura disponibilizados para consecução dos objetivos deste trabalho.

\section{REFERÊNCIAS}

ABBASI, T.,ABBASI, S. A.: Water Quality Indices. Amsterdam, The Netherlands: Elsevier Science, 2012. 384 p. https://doi.org/10.1016/B978-0-444-54304-2.00001-4

ALMEIDA, R.A.S. Índice de qualidade de águas subterrâneas destinadas ao uso na produção de água potável, Salvador, BA. Dissertação (Mestrado) - Universidade Federal da Bahia, Escola Politécnica, 2007.

ALMEIDA R. A. S. Índice de qualidade de uso da água subterrânea (e- IQUAS): uma metodologia de modelagem numérica flexível. Tese (Doutorado). Salvador, 2012. 334 f.

ANA. Panorama da qualidade das águas superficiais do Brasil: 2012. Brasília: Agência Nacional de Águas, 2012. 264 p.

BOOTSTRAP (Usa). Build fast, responsive sites with Bootstrap. Disponível em: https://getbootstrap.com/. Acesso em: 21 jun. 2020.

BROWN, R.M., McCLELLAND, N. I., DEININGER, R. A.,TOZER, R.G. A Water quality index: Do we dare? Water and Sewage Works, v.117, n.10, p. 339-343, 1970.

CADILHAC, L. , ALBINET, M. (Coord). Système d'évaluation de la qualité des eaux souterraines: rapport de présentation, Version 0 , Agences de l'eau et le Ministère de 
l'Ecologie et du Développement Durable, France, 2003. Disponível em:

http://oai.eauetbiodiversite.fr/entrepotsOAl/ElA/B16751.pdf. Acesso em: 26 Jul. 2020

CANADA. CANADIAN COUNCIL OF MINISTERS OF THE ENVIRONMENT CCME.

Canadian environmental quality guidelines: calculators. Disponível em:

https://www.ccme.ca/en/resources/canadian environmental quality guidelines/calculators.ht ml. Acesso em: 20 ago. 2020

CECCONELLO, Samanta Tolentino; CENTENO, Luana Nunes; GUEDES, Hugo Alexandre Soares. Índice de qualidade de água modificado pela análise multivariada: estudo de caso do Arroio Pelotas, RS, Brasil. Eng. Sanit. Ambient., Rio de Janeiro , v. 23, n. 5, p. 973978, Oct. 2018. Disponível em:

http://www.scielo.br/scielo.php?script=sci arttext\&pid=S1413-

41522018000500973\&lng=en\&nrm=iso. Acesso em: 21 ago. 2020.

https://doi.org/10.1590/s1413-41522018165394.

CETESB - Companhia de Tecnologia de Saneamento Ambiental, São Paulo Apêndice D Índices de qualidade das águas. São Paulo: CETESB, 2017 Disponível em https://cetesb.sp.gov.br/aguas-interiores/wp-

content/uploads/sites/12/2017/11/Ap\%C3\%AAndice-D-\%C3\%8Dndices-de-Qualidade-das\%C3\%81guas.pdf. Acesso em 12 jan. 2020.

FERREIRA, Kássia Crislayne Duarte et al. Adaptação do índice de qualidade de água da National Sanitation Foundation ao semiárido brasileiro1. Rev. Ciênc. Agron., Fortaleza, v. 46, n. 2, p. 277-286, June 2015. Disponível em:

http://www.scielo.br/scielo.php?script=sci arttext\&pid=S1806-

66902015000200277\&lng=en\&nrm=iso. Acesso em: 21 ago. 2020.

https://doi.org/10.5935/1806-6690.20150007

FSF FREE SOFTWARE FOUNDATION (Boston (usa)) (org.). Free software is software that gives you the user the freedom to share, study and modify it. We call this free software because the user is free. 2020. Free Software Foundation. Disponível em: https://www.fsf.org/about/what-is-free-software. Acesso em: 20 jun. 2020.

INSTITUTO MINEIRO DE GESTÃO DAS ÁGUAS. Séries Históricas de Monitoramentos da Qualidade das Águas Superficiais no Estado de Minas Gerais. Belo Horizonte: Igam, 2012-2016. Planilha. Disponível em http://200.198.57.118:8080/jspui/handle/123456789/414 Último Acesso: 11.Mai.2020

INSTITUTO MINEIRO DE GESTÃO DAS ÁGUAS. Médio e Baixo Jequitinhonha (UPGRH JQ3): panorama de qualidade das águas superficiais: primeiro trimestre de 2016. Belo Horizonte: Igam, 2016. 1 mapa. Escala: 1:1.200.000. Disponível em http://200.198.57.118:8080/jspui/handle/123456789/1964. Acesso em: 11 maio 2020.

JQUERY (NYC (USA)). What is jQuery? Disponível em: https://jquery.com/. Acesso em: 21 jun. 2020.

LARAVEL (Cramerton (usa)). The PHP Framework for Web Artisans. Disponível em: https://laravel.com/. Acesso em: 21 jun. 2020.

MYSQL. MySQL Community Server. Disponível em:

http://www.mysql.com/downloads/mysql/ Acesso em: 10 Jan 2011.

MySQL Workbench. Disponível em: http://www.mysql.com/products/workbench/. Acesso em: 12 maio 2012. 
RUMBAUGH, J.; JACOBSON, J.; BOOCH, G. The unified modeling language reference manual, Addison Wesley, 2nd edition, 2004

SILBERSCHATZ, A., KORTH, H. and SUDARSHAN S., Database system concepts , Sixth Edition, McGraw Hill Book Co., 2010

UNESCO. Relatório mundial das nações unidas sobre desenvolvimento dos recursos hídricos: soluções baseadas na natureza para a gestão da água: sumário executivo, 2019. Disponível em: https://unesdoc.unesco.org/ark:/48223/pf0000367303 por Acesso em 30.Abr.2020

WALKER, Aaron. Open-Source and Free Database Software. 2017. Disponível em: https://learn.g2.com/free-database-software. Acesso em: 5 dez. 2017

WATER RESEARCH CENTER (USA). Monitoring the quality of surface waters (WQI Calculator). Disponível em: https://www.water-research.net/index.php/water-treatment/watermonitoring/monitoring-the-quality-of-surfacewaters. Acesso em: 20 ago. 2020 